Musées, Patrimoine et Culture scientifiques et techniques

$126 \mid 2009$

novembre - décembre 2009

\title{
Le Vaisseau : De l'évaluation à la démarche qualité
}

Testimonials of evaluation practices

Isabelle Süss

URL : http://journals.openedition.org/ocim/218

DOI : 10.4000/ocim.218

ISSN : 2108-646X

Éditeur

OCIM

Édition imprimée

Date de publication : 1 novembre 2009

Pagination : 49-52

ISSN : 0994-1908

Référence électronique

Isabelle Süss, "Le Vaisseau : De l'évaluation à la démarche qualité », La Lettre de l'OCIM [En ligne],

126 | 2009, mis en ligne le 01 novembre 2011, consulté le 03 mai 2019. URL : http://

journals.openedition.org/ocim/218; DOI : 10.4000/ocim.218 


\title{
Le Vaisseau
}

\section{De l'évaluation à la démarche qualité}

\author{
Isabelle Süss *
}

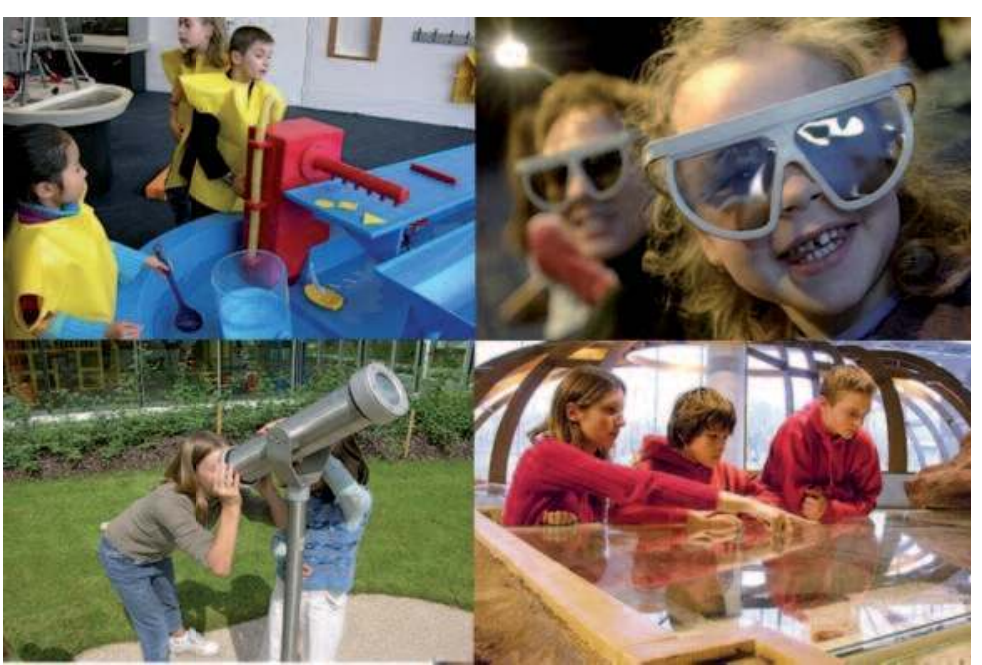
Le Vaisseau : découvrir, apprendre, s'amuser...
๑) Jean-Luc Stadler

* Isabelle Süss est chargée de l'Évaluation et de la Planification au Vaisseau isabelle.suss@cg67.fr
Le Vaisseau, équipement éducatif du Conseil général du Bas-Rhin, a ouvert ses portes en février 2005 à Strasbourg. C'est un lieu de découverte des sciences et des techniques, qui s'adresse en priorité aux 3/15 ans. Il constitue pour les parents et les enseignants un outil éducatif et interactif dont le concept est illustré par le slogan «Apprendre en s'amusant ».

\section{Une gestation sous haute surveillance}

Les étapes qui jalonnent la naissance du projet du Vaisseau sont nombreuses et placées sous le signe d'études préparatoires rigoureuses et détaillées. En effet, le Conseil général, porteur du projet, a demandé en 1999 une première étude de faisabilité pour la création d'un «Pôle de découverte de la culture scientifique et des techniques à destination des enfants et adolescents ». Le principe est adopté par les élus du département en 2000 et un comité de pilotage se met immédiatement en place. Le projet est lancé. En 2002, une étude complémentaire précise le cadre de la programmation culturelle et de l'aménagement du jardin.

Avant même ses fondations, les fées de l'évaluation se sont penchées sur le berceau du futur Vaisseau. Début 2003, une étude qualitative de perception s'est attachée à comprendre les attitudes, comportements et attentes des familles avec enfants (françaises et allemandes). Les discussions avec 19 parents et grandsparents réunis en 2 tables rondes ont permis de tester le principal parti pris du projet, et notamment :

- les thèmes,

- l'attractivité du concept ainsi que l'intérêt du positionnement ludique et participatif,

- la localisation géographique et la dénomination.

De cette étude a également été déduit un potentiel de visite, assorti d'éventuels moyens de l'améliorer. 


\section{La phase projet}

Forte de ces résultats, la phase projet a été abordée avec assurance, tout en poursuivant les réflexions engagées pour l'ouverture au public. L'équipe projet s'est entourée d'un comité scientifique et pédagogique, afin d'asseoir la réputation et le sérieux des activités développées au Vaisseau. Ces experts, issus des domaines de la médiation des sciences et des techniques, se sont réunis pour la première fois en 2004.

L'ambition de l'équipe projet de garantir la qualité des offres et services l'a amenée à envisager toutes les situations pour répondre avec pertinence et efficacité dans les meilleurs délais. Une attention particulière a été portée :

- à la gestion des risques par une analyse réaliste,

- à l'accueil des publics par la mise en place de protocoles d'accueil détaillés,

- à la gestion d'équipe par la définition de valeurs communes.

Pour certains de ces travaux, le Vaisseau s'est appuyé sur les compétences de consultants, spécialisés pour l'un dans la gestion des publics dits « à risque » (dans le cadre du Vaisseau il s'agit des jeunes, essentiellement ados et pré-ados, dont le comportement en groupe pourrait entraîner débordements et/ou dégâts matériels) et pour le second dans le management.

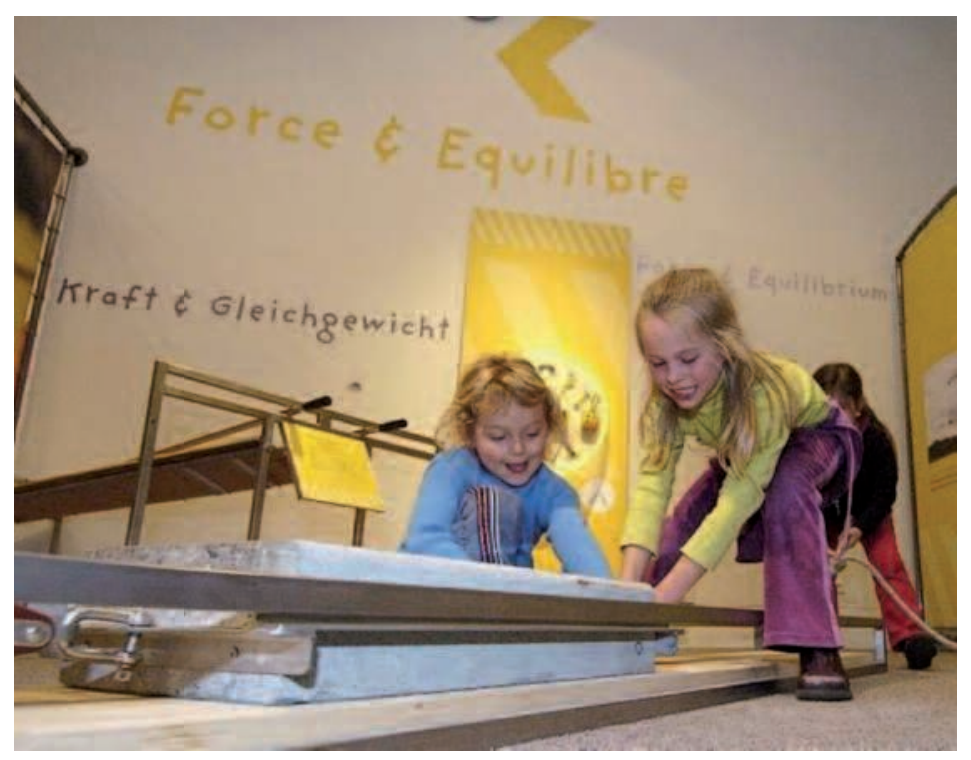

Au vaisseau, il est interdit de ne pas toucher: jeu et apprentissage sont intimement liés. (c) Jean-Luc Stadler
Cette volonté de ne rien laisser au hasard a conduit à l'ultime étape avant l'inauguration officielle : l'observation en situation réelle de plusieurs catégories de visiteurs pendant la «période à blanc».

Dès les prémices du projet, la prise en compte des situations de handicap a fait partie intégrante des réflexions de fond. C'est tout naturellement qu'une journée d'observation a été programmée afin de déceler les dysfonctionnements et améliorer le confort et l'accueil de ces visiteurs : 150 enfants de centres spécialisés et de handicaps différents (moteurs, mentaux, malentendants et malvoyants) ont pu tester l'accessibilité des lieux et des éléments.

Les enseignants ont à leur tour fait l'objet d'une étude au travers d'un questionnaire approfondi mis à leur disposition : 123 enseignants ont donné leur avis, tant sur les aspects pédagogiques que sur les considérations pratiques et logistiques de l'organisation d'une visite scolaire du Vaisseau.

Les visiteurs individuels ont eux aussi été invités à tester en famille l'accueil, les installations et les procédures. L'évaluation détaillée de leur visite a contribué à parfaire les offres et les services du Vaisseau, quelques jours avant l'ouverture officielle.

Les premiers visiteurs ont été accueillis le 22 février 2005, avec une accessibilité totale à tous les éléments du Vaisseau.

\section{La politique d'évaluation}

La question de l'évaluation de terrain est posée dès la phase projet. Les démarches prospectives, initiées dès l'origine du projet, ont imprimé la volonté de pérenniser une vigilance au quotidien, avec l'ambition de « dresser une cartographie des points forts et des points faibles de l'équipement » (1). Toutes les compétences et activités de l'équipement sont concernées, de la maintenance technique aux offres culturelles, des activités commerciales à la satisfaction des visiteurs. Chaque service a mis en place ses propres outils de contrôle, avec pour objectif de «mettre le visiteur et sa satisfaction au cœur du Vaisseau » (2). Les exemples qui suivent illustrent l'état d'esprit de la démarche :

- un suivi des horaires d'ouverture et de fermeture des portes du Vaisseau a permis d'identifier les premières causes de retard et d'envisager les solutions appropriées ;

- des outils de comptabilité analytique, de suivi budgétaire et de gestion du temps de travail permettent une maitrise rigoureuse des moyens financiers et humains ; - les activités commerciales sont cadrées par des objectifs revus annuellement ; 
- la maintenance technique a construit un outil de suivi des pannes et inconforts des éléments du Vaisseau, avec pour objectif de ne pas dépasser $3 \%$ de pannes ;

- un agenda 21, adapté au Vaisseau et à ses contraintes, a été rédigé et validé par le Conseil général ;

- enfin, chaque offre culturelle est envisagée comme une bonne occasion " pour observer, faire le bilan, comprendre, apprendre et avancer... (3).

Afin de définir et cadrer la politique d'évaluation des offres culturelles, le Vaisseau s'est d'abord fait assister par un consultant qui a remis quelques mois après l'inauguration du Vaisseau, un guide méthodologique. C'est sur ces premières bases, et dans le cadre de travaux de recherche, qu'ont été réalisées les premières enquêtes et observations auprès des visiteurs du Vaisseau. Et notamment à l'occasion de programmations événementielles, comme la Fête de la science ou l'Année mondiale de la Physique. Toutes ces données alimentent largement le bilan détaillé de la première année de fonctionnement du Vaisseau, qui présente en conclusion 10 axes de progrès.

\section{L'évaluation des offres et des visiteurs : une stratégie à l'épreuve du terrain}

Face à l'affluence des visiteurs dès les premiers mois d'ouverture, l'équipe en place s'est réorganisée, privilégiant les actions de terrain et une gestion très

\section{Chronologie de la démarche d'évaluation au Vaisseau}

1999 : Étude de faisabilité d'un «Pôle de découverte de la culture scientifique et des techniques à destination des enfants et adolescents »

2000 : Mise en place d'un comité de pilotage

2002 : Étude complémentaire pour la programmation culturelle et le jardin

2003 : Étude qualitative de perception d'un « Pôle de découverte de la culture scientifique...»

2004 : Première réunion du comité scientifique du Vaisseau

Janvier 2005 : Observation des publics en situation de handicap

Février 2005 : Évaluation auprès des enseignants

Février 2005 : Évaluation auprès des visiteurs familiaux pendant la «période à blanc »

\section{2 février 2005 : Ouverture du Vaisseau}

Mai 2005 : Guide méthodologique de l'évaluation Juin 2005 : Bilan de l'observation des familles Décembre 2005 : Bilan de la Fête de la science et de l'Année mondiale de la Physique

Décembre 2005 : Étude de notoriété et d'image

\section{6 : Bilan de la première année} de fonctionnement du Vaisseau

10 axes de progrès dont la « Mise en œuvre des réponses aux besoins identifiés lors des évaluations »

\section{7 : Travaux de recherche dans le cadre d'une thèse universitaire}

- Évaluation par le visiteur des apports pédagogiques et linguistiques du Vaisseau

- Observations dans les expositions permanente et temporaire

\section{Septembre 2007 : Création d'un poste dédié à l'évaluation des offres et des publics}

\section{8 : Mise en place de la stratégie d'évaluation}

- Évaluation quantitative : suivi et analyse de la fréquentation annuelle, mensuelle et journalière pendant et hors vacances scolaires

- Mise en place d'une démarche qualité et de différentes enquêtes qualitatives

Janvier 2008 : Bilan qualitatif de l'événement Année

Polaire internationale

Février 2008 : Enquête de perception de l'exposition À Table, doublée d'une enquête en interne Avril et été 2008 : Enquête auprès des visiteurs de l'exposition temporaire Dialogue dans le Noir et initiation d'une démarche qualité via des " visiteurs mystère » Juin 2008 : Bilan de l'enquête en phoning auprès des enseignants

Juillet/août 2008 : Enquête sur le Jardin du Vaisseau Août 2008 : Cartographie de l'origine géographique des visiteurs du Bas-Rhin

Octobre à décembre 2008 : Évaluation qualitative de l'exposition-événement Enquête de Maths

Novembre 2008 : Bilan qualitatif des Forums collégiens Décembre 2008 : Lancement de l'enquête de notoriété (phase 1)

Février 2009 : Évaluation des stages

«Mission martienne»

2009 : Bilan de la première année d'évaluation

«Évaluation et démarche qualité au Vaisseau » 


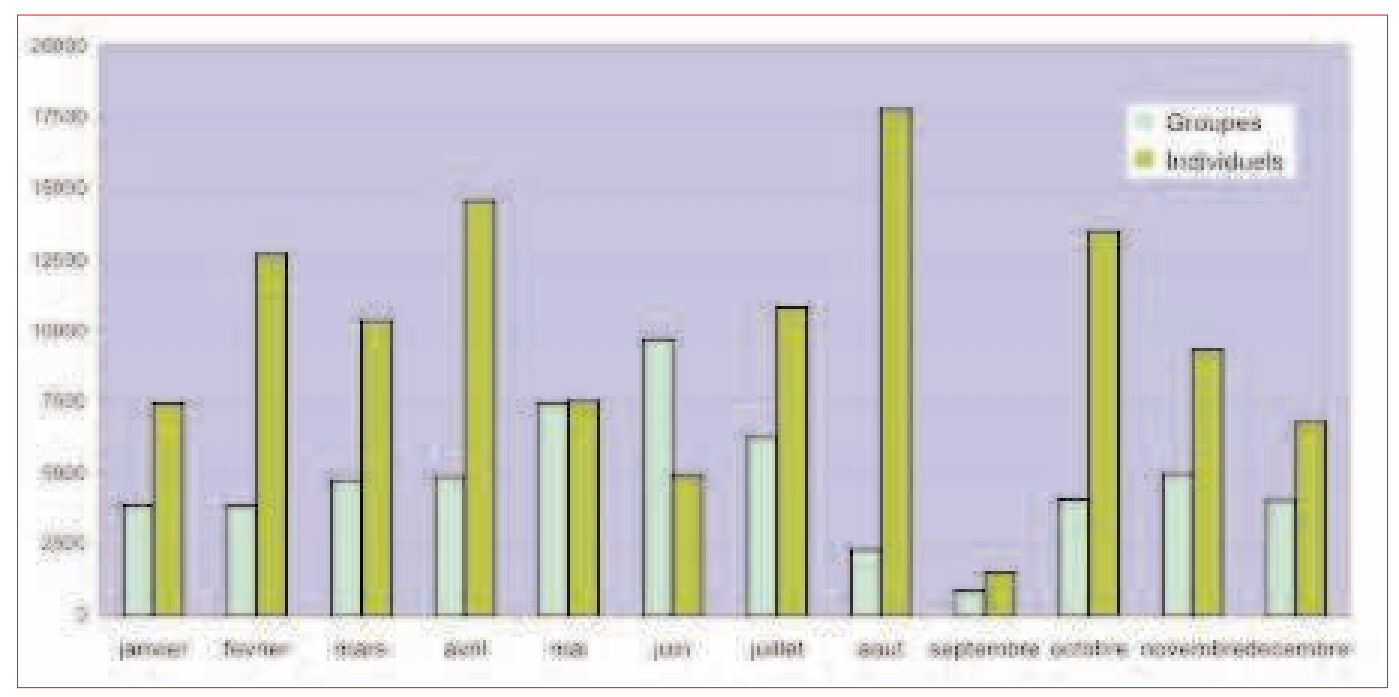

Cycle de fréquentation en 2008 des 2 principaux publics du Vaisseau :

les groupes (essentiellement scolaires) et les individuels (ou familles)

opérationnelle. Sans pour autant le perdre de vue, l'axe de progrès concernant l'évaluation a été momentanément mis en veille.

Après deux années et demie de fonctionnement, à un régime bien supérieur aux prévisions, le choix d'un poste permanent dédié à l'évaluation s'est imposé comme une évidence.

Dans le prolongement des études préparatoires et des travaux de recherche sur les premiers visiteurs, la fonction «Évaluation du Vaisseau » s'est mise en place avec l'ambition de proposer des outils pratiques et utiles au quotidien pour améliorer les activités respectives des différents services. La priorité a été donnée à l'évaluation des publics et des offres.

Un premier volet d'études a consisté à analyser la fréquentation sous divers angles, afin d'en extraire les cycles de fréquentation selon les publics et les périodes de l'année. Fréquentation mensuelle, fréquentation journalière moyenne, fréquentation aux différentes périodes de vacances scolaires sont autant de paramètres pour suivre au plus près l'évolution des entrées au Vaisseau. Le cycle annuel de fréquentation du Vaisseau est aujourd'hui connu, autant pour les visiteurs individuels que pour les scolaires (voir graphique). Un cycle hebdomadaire, qui varie selon les périodes de l'année a également été mis en évidence. La fréquentation des visiteurs allemands est en cours d'étude, afin d'intégrer judicieusement les offres bilingues. Ces données sont utilisées par le Service culturel pour ajuster au mieux la programmation et adapter les moyens humains à la fréquentation attendue. Ces chiffres servent à l'élaboration pour les visiteurs d'un calendrier indicatif des périodes rouges (de très fortes fréquentations) et des périodes oranges ou vertes (de moindre fréquentation).
Pour compléter ces données chiffrées, diverses études qualitatives ont été menées au travers d'enquêtes auprès des visiteurs. Les résultats reflètent la satisfaction et les attentes des visiteurs et permettent de suivre au plus près la qualité des prestations du Vaisseau. S'y ajoutent les indicateurs spécifiques, sélectionnés pour suivre l'évolution des publics, et régulièrement mis à jour : le taux de primo-visiteurs, l'origine géographique, la pyramide des âges des enfants, les canaux de communication, la notoriété... Ces informations capitales sur le comportement des visiteurs, qu'ils soient scolaires ou familiaux, viennent alimenter les débats de fond sur les grandes orientations de gestion du Vaisseau et éclairer les choix stratégiques à moyen et long terme.

Le champ des évaluations est encore vaste. Cependant, chaque évaluation n'est validée qu’à la condition que les précédents résultats aient été pris en compte et exploités. À cet égard, l'ensemble des travaux d'évaluation ont été regroupés et présentés de manière synthétique dans le document «Évaluation et démarche qualité au Vaisseau ». Ce bilan est autant une vitrine des activités d'évaluation du Vaisseau, qu'un guide à utiliser au quotidien pour le personnel. Il sera présenté à chaque service, afin de s'assurer que le personnel, déjà familiarisé avec la démarche, s'approprie au mieux les résultats.

\section{Notes}

(1) et (2) Note interne, août 2004

(3) Bilan de la première année de fonctionnement 